\title{
Expresión de la sexualidad de una habitante de una residencia terapéutica como ocio: encuentros entre Elias y Dunning y la Terapia Ocupacional
}

The expression of a therapeutic resident's sexuality as leisure: Encounters between Elias \& Dunning and Occupational Therapy

A expressão da sexualidade de uma moradora de residência terapêutica como lazer: encontros entre Elias \& Dunning e a Terapia Ocupacional

Adriana Gonçalves Queiroz ${ }^{1}$

Ana Cláudia Porfírio Couto ${ }^{2}$

Ludimila Canário Silva Barreto ${ }^{3}$

Cristiane Miryam Drumond de Brito ${ }^{4}$

Recibido: 26 de junio 2019 • Enviado para modificación: 23 de julio 2020 • Aceptado: 8 de agosto 2020

Queiroz, A.G., Couto, A.C.P., Barreto, L.C.S. y Brito, C.M.D. (2020). Expresión de la sexualidad de una habitante de una residencia terapéutica como ocio: encuentros entre Elias y Dunning y la Terapia Ocupacional. Revista Ocupación Humana, 20 (1), 106-117. https://doi.org/10.25214/25907816.263

\footnotetext{
1 Terapeuta ocupacional. Postgrado lato sensu en Intervención Psicosocial en el Contexto de las Políticas Públicas. Magíster y doctoranda en Estudios Interdisciplinarios del Ocio, Universidad Federal de Minas Gerais. Belo Horizonte, Brasil. adrianaqueiroz.to@gmail.com iD https://orcid.org/0000-0002-0634-9022 W

${ }^{2}$ Licenciada en Educación Física. Máster en Educación Física. Doctora en Ciencias del Deporte. Postdoctorado en Sociología del Deporte y el Ocio. Docente, Universidad Federal de Minas Gerais. Belo Horizonte, Brasil. acpcouto@gmail.com iD https://orcid.org/0000-0003-3457-0987

${ }^{3}$ Terapeuta ocupacional. Postgrado lato sensu en Gestión de Recursos Humanos. Especialista en Salud Mental. Estudiante de Maestría en Estudios en Ocupación, Universidad Federal de Minas Gerais. Servicio de Residencias Terapéuticas. Belo Horizonte, Brasil. ludicsilva@gmail.com iD https://orcid. org/0000-0002-0942-5975

${ }^{4}$ Terapeuta ocupacional. Magíster y doctora en Comunicación y Semiótica. Docente, Universidad Federal de Minas Gerais. cdrumonddebrito@gmail.com iD https://orcid.org/0000-0002-2802-2119
} 


\title{
RESUMEN
}

La reforma psiquiátrica brasileña, además de un modelo de cuidado para las personas con trastornos de salud mental, presentó también una nueva manera de entender sus derechos y su modo de vida. En este artículo, a partir de la entrevista a una moradora de una residencia terapéutica de Belo Horizonte, Brasil, se realiza un análisis sobre el ocio a la luz del conocimiento de los autores de las Ciencias Sociales Elias y Dunning, quienes hablan de excitación y placer en el ocio, y se plantean algunas implicaciones para Terapia Ocupacional. Es posible comprender las vivencias de deseo sexual de la residente como formas de vivir el ocio que ejercen una función en su convivencia con la sociedad y resignifican sus posibilidades de probar, inventar y reinventar el ocio y la cotidianidad en su nuevo rol.

\section{PALABRAS CLAVE}

ocio, salud mental, autonomía personal, sexualidad, Terapia Ocupacional

\begin{abstract}
In addition to a model of care for people with mental health disorders, the Brazilian psychiatric reform also presented a new way of understanding their rights and way of life. In this article, based on an interview with a resident of a therapeutic residence in Belo Horizonte, Brazil, an analysis of leisure is carried out based on the knowledge of Elias and Dunning, Social Science authors, who discuss excitement and pleasure in leisure. Subsequently, some implications for Occupational Therapy are raised. It is possible to understand the resident's experiences of sexual desire as ways of living through leisure. Sexual desire has a function in her coexistence with society and redefines her possibilities of trying, inventing, and reinventing leisure and everyday life in her new role.
\end{abstract}

\section{KEY WORDS}

leisure, mental health, personal autonomy, sexual behavior, Occupational Therapy

\section{RESUMO}

A reforma psiquiátrica brasileira, além de um modelo de cuidado às pessoas com transtornos psiquiátricos, também apresentou uma nova maneira de ver os direitos e o modo de vida das pessoas. Neste artigo, a partir de uma entrevista semiestruturada, realizada com uma moradora de residência terapêutica em Belo Horizonte, Brasil, foi elaborada uma análise sobre o lazer, à luz do conhecimento de autores das Ciências Sociais, Elias e Dunning (1992). Os autores falam de excitação e prazer no lazer e algumas implicações para a Terapia Ocupacional são destacadas. É possível compreender as experiências de desejo sexual da moradora como formas de viver o lazer. Tais formas exercem uma função na sua convivência com a sociedade e ressignificam suas possibilidades de experimentar, inventar e reinventar o lazer, bem como o cotidiano em seu novo papel.

\section{PALAVRAS-CHAVE}

lazer, saúde mental, autonomia pessoal, sexualidade, Terapia Ocupacional 


\section{Introducción}

Los servicios de hogares terapéuticos, también conocidos como residencias terapéuticas, o simplemente como casas, son viviendas que reciben a pacientes procedentes de hospitales psiquiátricos durante el proceso de desinstitucionalización (Ordenanza Ministerial 106/2000). Se disponen para ser casas en la comunidad, vinculadas a la red de salud local.

En cumplimiento de su función como viviendas y potenciadoras del continuo proceso de desinstitucionalización, las residencias terapéuticas buscan estimular a la o el residente - expaciente de larga permanencia en un hospital psiquiátrico, paciente de la red de salud, habitante de la ciudad -, a (re)adquirir cada día mayor autonomía en sus actividades de la vida diaria, tanto básicas como instrumentales y avanzadas (Dias et al., 2011). En la práctica, esto significa que existe un intenso trabajo para que la persona se convierta cada vez más en una residente de la casa, a partir de su apropiación de este espacio, desde la elección de su habitación y enseres hasta la búsqueda de sus medicamentos en el centro de salud, la compra del pan en la panadería y la elección de sus actividades de ocio.

Para ayudarles en esa continua conquista, las y los residentes cuentan en la casa con un equipo conformado por cuidadores, practicantes y una supervisora. Este equipo tiene en la Ordenanza para la creación de las residencias terapéuticas (Ordenanza Ministerial 106/2000) el norte para sus acciones y para la cons- trucción de los proyectos terapéuticos de cada residente. Dichos proyectos deben incluir la asistencia en vivienda, salud, educación y seguridad social, entre otras, y promover su (re) inclusión en la comunidad, en todas las dimensiones posibles. Así, además del equipo, las y los residentes cuentan fuera de la casa con aquellas relaciones que construyen en la cotidianidad - con familiares, vecinos, agentes comunitarios en salud, comerciantes locales, etc.

Son los intercambios que acontecen en el diario vivir los que tejen y modelan la nueva cotidianidad de las personas que egresan de las instituciones psiquiátricas. Esa cotidianidad, o cotidiano, se comprende en este artículo como fuente social en la que cada microevento está marcado por rasgos culturales, retratos de las situaciones sociales de la vida en las cuales se crean vínculos sociales, emocionales y racionales (Covino, 2007; Salles y Matsukura, 2013). Es a partir de esos intercambios que la o el residente hace frente a la ansiedad que le genera aquel nuevo ambiente que no es hospital ni clínica; en su lugar, es una casa con todos los significados y significantes que ello implica. Las costumbres, dichos y hábitos manicomiales ${ }^{5}$ se dejan de lado o se reconfiguran, al tiempo que se adquieren nuevos hábitos y costumbres.

En las residencias terapéuticas, a sus habitantes se les informa que la casa es suya, por consiguiente, se les invita a asumir responsabilidades hacia esta. Como es una casa, no hay médicos,

\footnotetext{
${ }^{5}$ Se refiere a hábitos adquiridos en los años de reclusión en el hospital psiquiátrico, tales como comer en cuclillas, seguir rutinas marcadas por los horarios de comidas, pedir permiso para ir de una parte de la casa a otra, usar ropas con nombre, fumar colillas de cigarrillo, tomar café frío, entre otros.
} 
tampoco enfermeras ${ }^{6}$ en función clínica, pero sí hay cuidadores que cumplen jornadas de 12 horas de trabajo por 36 de descanso. Se agrega al equipo una practicante que tiene la misma formación académica de la supervisora. En Belo Horizonte, hasta el momento, existen supervisoras con formación en Enfermería, Psicología, Terapia Ocupacional y Trabajo Social. En la casa que se menciona en este artículo, la supervisora tiene formación en Terapia Ocupacional.

En este contexto, el ocio se inserta como una vivencia más en la construcción y la apropiación de la nueva cotidianidad, de la nueva vida y las nuevas relaciones. Por ser una dimensión de la cultura y estar representado por la voluntad propia de cada sujeto, la manera de experimentar el ocio, en ese proceso de construcción del rol como residente, es singular.

Reconociendo que no hay muchos estudios que aborden el tema del papel del ocio en estos procesos de desinstitucionalización y transformación de pacientes en residentes, este texto tiene como objetivo discutir con respecto a las elecciones de ocio de una de las habitantes de una residencia terapéutica de Belo Horizonte, Brasil, desde la perspectiva de los autores Nobert Elias y Erick Dunning y en relación con la Terapia Ocupacional.

$$
\text { Elias y Dunning (1992) son conocidos }
$$

en el campo de las Ciencias Sociales por estudiar la excitación y el placer en el ocio. Las implicaciones de esta perspectiva para la Terapia Ocupacional serán discutidas con algunos preceptos de la Ciencia Ocupacional, cuando esta considera la ocupación como forma, función y significado (Zemke y Clark,1996).

Queiroz (2015), primera autora de este artículo, en su tesis de Maestría en Estudios Interdisciplinarios del Ocio en la Universidad Federal de Minas Gerais, Brasil, buscó comprender las ocupaciones reconocidas como ocio y su importancia en el diario vivir de 17 habitantes de residencias terapéuticas de Belo Horizonte $^{7}$. Como parte de ese estudio, realizó una entrevista semiestructurada y observó desde su lugar como investigadora y supervisora de la residencia terapéutica a Frida ${ }^{8}$, una de las residentes. Las respuestas de Frida constituyeron el punto de partida para las discusiones que se condensan en este texto respecto a la autonomía y la sexualidad como ocio en el diario vivir, en la perspectiva de los autores Nobert Elias y Erick Dunning (1992).

\section{Frida, su llegada a la residencia}

Frida: Yo llegué aquí, a esta casa, (aquí a Sobral ${ }^{9}$ ), fue así. Ellos me dieron de alta allá.

${ }^{6}$ En casas en las que habitan residentes más dependientes puede contratarse una supervisora con formación en Enfermería, pero su papel en la casa es de gestión; puede ayudar en los cuidados, pero no prescribirlos o dirigirlos. Aunque hay cuidadores con formación técnica en Enfermería, no les corresponde ese tipo de cuidado. Cuando se necesiten cuidados de enfermería, esto debe comunicarse al centro de salud.

${ }^{7}$ La disertación de maestría fue presentada en 2015 bajo el título No estoy bien, necesito salir a pasear: el papel del ocio en el diario vivir de habitantes de residencia terapéutica de Belo Horizonte. Fue una investigación cualitativa, transversal, realizada en 2014.

${ }^{8}$ Nombre ficticio.

${ }^{9}$ Nombre ficticio de la casa. 
Entrevistadora: Allá, ¿dónde?

Frida: Allá en la Sagrada Señora de la Luz $^{\mathbf{1 0}}$. Entonces yo vine para acá. Llegué aquí y la primera cosa que yo hice fue elegir mi pieza. Elegí aquella pieza, elegí aquella pieza.

Entrevistadora: ¿Por qué elegiste esa pieza?

Frida: Yo quise la segunda pieza, o la tercera, yo no sé. Elegí aquella por el azulejo (...) entonces, yo elegí. La primera cosa que yo hice después de entrar a la pieza fue quemar mi foto...

\section{Entrevistadora: ¿Por qué quemaste tu foto $3 \times 4$ ?}

Frida: No sé, quemé, jesa casa retumba en mi cabeza!

Frida, de 55 años de edad, es proveniente de la región metropolitana de Belo Horizonte, tiene muchos hermanos y a su madre, su padre murió. Estuvo internada en una clínica psiquiátrica de Belo Horizonte por veinte años; como las demás personas que allí permanecían, antes de su salida, en el año 2008, recibió acompañamiento del equipo de desinstitucionalización del ayuntamiento de Belo Horizonte. En la clínica era conocida como la fugitiva, pues siempre huía de un ala a otra intentando, en realidad, escapar de la clínica. Esa búsqueda de libertad, mal interpretada dentro de los altos muros de ese establecimiento ubicado en una elegante zona de la ciudad, era su marca cuando estaba institucionalizada y siguió siéndolo después de su alta de la clínica.

El 26 de diciembre del 2008, Frida llegó a la residencia; un lugar que aún intentaba descifrar: ¿es una clínica?, ¿una casita ${ }^{11}$ ?, ¿una casa? ¿La casa de quién?, ¿mí casa? Pero... ¿es mi casa con mi familia? ¿Dónde está el Doctor?, ¿la enfermera? ¿Yo puedo elegir? ¡Yo tengo poder de elección! Algo que realmente puede desorientar.

En la casa viven seis hombres y cuatro mujeres, incluida ella. Frida eligió a una vieja amiga de la clínica como compañera de habitación. Como en cualquier casa, existe una rutina - desayunar, tomar la medicación, ducharse, comprar pan, limpiar, cuidar sus pertenencias, dialogar, ver la televisión, Ilamar a amigos o familiares por teléfono, salir un rato, etc. - , pero a diferencia de la rigidez del hospital, a sus residentes no se les obliga a ducharse en la mañana, a comer en horarios específicos, ni a dormir a una hora establecida.

\section{De (im)paciente a moradora: la sexualidad como ocio en la cotidianidad}

Cinco días después de llegar a la casa, Frida repitió un hábito que había adquirido en el manicomio ${ }^{12}$, saltó las rejas del pa-

10 Nombre ficticio del establecimiento psiquiátrico en el que Frida permaneció. Las clínicas y los hospitales psiquiátricos tenían las mismas reglas, pero menor infraestructura física, en el caso de las primeras. Por los hábitos que allí se mantenían, a ambos se les llamaba manicomios. El término clínica, en este texto, se usa para hacer referencia al servicio hospitalario de cual Frida salió. El término hospital u hospitalario se refiere al cuidado en general. Además, el término manicomio se emplea para denominar el lugar que mantenía hábitos y cultura de exclusión y violencia.

11 Término utilizado, entre el personal de psiquiatría, para el lugar en el que se realizaba terapia electroconvulsiva a las y los internos.

12 Manicomio es una institución cerrada que busca normatizar a los individuos, dominarlos y ejercer su poder al institucionalizar y desconocer su subjetividad (Brito y Dimenstein, 2008). 
tio y salió a las calle. Exactamente como la madre de Frida lo había dicho: - ¿ustedes creen que esa reja detendrá a Frida? ;Tengan cuidado, ella va a huir! - Claramente, la familia también estaba institucionalizada.

En definitiva, si Frida escapaba del ala principal del manicomio, ¿no huiría de una simple casa? Pero así como ella lo hizo, poco a poco su familia también entendió que las rejas del patio no estaban allí para aprisionar y que, en la casa, las y los residentes ejercían su derecho de ir y venir libremente. Ella ya no era una (im)paciente que daba trabajo al personal del hospital por sus constantes escapes, sino una moradora en construcción.

Fueron innumerables fugas, algunas veces Frida pasaba varios días afuera y en otras ocasiones volvía en los horarios que ella misma organizaba, generalmente, a las 9:00 a.m. del día siguiente. Cada vez, Frida sorprendía al equipo con los relatos de sus aventuras: había hecho autostop, sabía exactamente el camino que había recorrido, hasta sabía cuándo era necesario ir al centro de salud, pues había tenido relaciones sexuales con habitantes de la calle sin usar preservativo. Siempre compartía sus vivencias de manera detallada, incluso algunas veces parecía tener la intención de incomodar a sus oyentes, pero generalmente parecía hacerlo por consideración con el equipo de la casa, quienes se preocupaban y ponían en acción toda la red de salud, asistencia social y policía para cuidarla.

Después de dos años viviendo de esa manera, en los que otras cuestiones de adaptación a la casa y a la nueva vida fueron tomando forma (como la relación con el centro de salud y la participación en la casa), y siguiendo el propósito de las residencias terapéuticas, explícito en su ordenanza de reinserción social, el equipo le entregó a Frida la llave de la casa. Su actitud, así como el lenguaje del equipo fueron cambiando; de manera que ya no eran fugas, sino salidas. Quien huye, huye de algo o de alguien. Finalmente, solo se puede huir de un lugar en el que se está atrapada; no era ese el caso de Frida. Quizás, hoy en día, se podría reemplazar la palabra salidas por encuentros, pues en cada salida Frida siempre encontraba alguna cosa, o a alguien, o a ella misma.

Tener la llave de la casa tornó en oficial, de alguna manera, que aquel espacio era suyo. Frida empezó a traer pretendientes a su morada, a su habitación. Otra mediación se iniciaba, pues el cuarto no era solo de ella, y tampoco la casa. ¿Cómo veían eso las demás personas que habitaban la residencia? Frida se encontraba en una situación complicada, ya no quería tener relaciones sexuales en la calle o en terrenos baldíos, pero sus compañeros y compañeras no aceptaban a personas desconocidas en la casa y se molestaban con las expresiones sexuales al interior de esta.

Antes, Frida dejaba la puerta abierta al cambiarse de ropa o al masturbarse (su habitación se encontraba en el pasillo entre el salón y la cocina), o usaba escotes profundos que dejaban a la vista el sostén, incluso a veces uno de sus senos. Provocaba sexualmente de manera (in)consciente a otros residentes de la casa, pero les desdeñaba para cuestiones importantes. Frente a los impases que se presentaban, empezó a prestarle atención a las pretensiones de dos residentes, pero aunque satisfacían 
sus deseos, no quiso sostener con ninguno de ellos una relación duradera.

En ese contexto, el equipo comenzó a preguntarse sobre el papel de la sexualidad para Frida. A través de la entrevista realizada y de la observación, se empezó a comprender que sus vivencias de deseo sexual se asociaban con su manera de experimentar, inventar y reinventar su ocio y a sí misma.

\section{Ocio y excitación: excitación en el ocio}

Entrevistadora: Frida, cuando te pregunto qué es ocio, busco saber qué te gusta hacer y qué te hace feliz.

Frida: ¿Qué me hace feliz?

Entrevistadora: Sí.

Frida: Me hace feliz... meter ${ }^{13}$ [susurra].

Entrevistadora: ¿Meter te hace feliz?

Frida: ;Ah, no importa! Yo chupo el pene de Jaime ${ }^{14}$ [susurrando].

Entrevistadora: ¿Qué? ¿Chupa?

Frida: El pene de Jaime [dice más fuerte].

Entrevistadora: El pene de Jaime, ¿eso te hace feliz?

Frida: ¡Ah, no importa! Y él chupa mi vagina.
"Los individuos que reaccionan de manera bastante excitada están sujetos a ser conducidos a un hospital o a la cárcel" (Elias y Dunning, 1992, p.102). La sociedad busca constantemente controlar el comportamiento de aquellas personas a las que Ilama diferentes; así, las excitaciones intensas se interpretan como descontrol, pues salen de los patrones aceptados como normales. A lo largo de la historia han existido innumerables intentos de control: reclusión de las locas y los locos en manicomios, que se fueron configurando como prisiones e institucionalizaron subjetividades; institucionalización de niños, niñas y jóvenes en fundaciones estatales para el bienestar de los menores -FEBEM ${ }^{15}$; aumento de la cantidad de prisiones y comunidades terapéuticas.

De esa manera, cuando Frida considera sus actividades sexuales como ocio, ella contradice el control que se espera que la sociedad imponga a una persona con un trastorno mental; denuncia que, aunque sea usuaria de servicios de salud mental y haya estado internada por muchos años, tiene deseos y se hace responsable por ellos.

En el ámbito de esta discusión, el papel del equipo de la residencia terapéutica es también el de ser mediador, que escucha y valida la manera en que el sujeto expresa su excitación como vivencia del ocio. Así, el ocio expresado por Frida, además de ser algo inherente al ser humano, un ser sexual y con sexualidad, es algo que "proporciona la esfera de acción para liberarse, de manera equilibrada, de las restricciones" (Elias y Dunning,

\footnotetext{
${ }^{13}$ Forma peyorativa de referirse a la actividad sexual.

${ }^{14}$ Nombre ficticio.

${ }^{15}$ Instituciones de acogida para adolescentes con propósitos de reparación de los efectos de la marginalidad, asistencia, corrección o castigo a menores infractores (Marazina, 2000).
} 
1992, p.105), en este caso, civilizadoras y estigmatizantes.

Más allá del contenido del ocio de Frida (actividades que proporcionan satisfacción sexual), es importante resaltar que ella lo interpreta como algo que la hace feliz. En ese sentido, Elias y Dunning hablan de la satisfacción que proporciona el ocio y de la importancia de este "para el bienestar de las personas como individuos y como sociedad" (1992, p.105). Así, hay un largo camino por recorrer para lograr ese bienestar individual y social, especialmente si se trata de grupos estigmatizados. Las restricciones impuestas a estas personas están enraizadas en la sociedad, de manera que sus actos apasionados son leídos como devaneos, no como actuaciones legítimas de sujetos en busca de sí mismos y en defensa de sus deseos y derechos. Elias y Dunning resumen bien cuando dicen que la excitación espontánea y elemental ha sido interpretada, probablemente, como "una enemiga de la vida humana en sociedad" (1992, p.113).

Estos autores, de cierta manera, retoman este tema cuando hablan de placer y ocio. El placer es con frecuencia eliminado de las conversaciones importantes $y$, ya que en una sociedad capitalista, como la actual, la productividad es lo que tiene valor, las actividades de ocio se convierten en secundarias, principalmente cuando están relacionadas con el placer que proporcionan a las personas; esas actividades, como el ocio de Frida, pasan a ser un tabú ${ }^{16}$.

Aunque la mirada del otro, muchas veces moralista, rodee la experiencia de Frida, se percibe que ella busca cierta tensión. Realiza sus actividades sexuales cuando la cuidadora no está o de manera rápida, pero con frecuencia lo hace en lugares de alta circulación, en los que puede ser vista o descubierta, como en la lavandería. Según lo resaltado por Elias y Dunning, el ocio puede generar y ser fuente de liberación de tensiones; de esta manera, se producen "tensiones de un tipo particular, el desarrollo de una agradable tensión-excitación como pieza clave de satisfacción en el ocio" (1992, p.136).

Los encuentros de Frida, sean fuera o dentro de la casa, parecen ser además una búsqueda de excitación, de evasión del deseo sexual, una ruptura en su rutina en la que "las actividades de ocio proporcionan, por un corto tiempo, la emergencia de sentimientos agradables y fuertes que, con frecuencia, están ausentes" (Elias y Dunning, 1992, p.137). Esa ruptura de la rutina es una decisión; así lo manifiesta:

Entrevistadora: Frida, ¿cómo decides qué hacer para tu ocio?

Frida: Es difícil.

Entrevistadora: ¿Es difícil?

Frida: Algunas veces tenemos hambre, entonces... es una decisión.

Entrevistadora: Es una decisión, ¿pero es ocio?

Frida: Sí.

Elias y Dunning reflexionan que, en algunas ocasiones, las personas no se dan cuenta de la importancia de su pro-

\footnotetext{
${ }^{16}$ Un sistema de supersticiones relacionadas con valores morales que normalmente se clasifican y aceptan socialmente como prohibidas (Orsi, 2011).
} 
pio placer y satisfacción en la elección de las actividades de ocio, pues estos no siempre provienen de otra persona, sino del sujeto mismo. Este postulado dialoga con el caso presentado, ya que Frida deja clara "la consideración de su propio placer, de su propia satisfacción" (1992, p.140) y toma la decisión de obtenerlos.

A veces incomoda a la sociedad, en otros momentos se adecúa a los limites socialmente establecidos (cuando usa condones o elige a un compañero conocido y no a un extraño, por ejemplo); pero tiene las decisiones en sus manos, en pleno ejercicio de su autonomía respecto a cómo vivir el ocio con su propio cuerpo. Sus experiencias de ocio, como se ejemplifica a continuación, hacen temblar el moralismo controlador que la sociedad intenta imponer.

\section{Entrevistadora: Frida, cuéntame una experiencia de ocio.}

Frida: Experiencia... mmm..., es difícil para mí... Dormir.

\section{Entrevistadora: ¿Dormir es una expe- riencia de ocio?}

Frida: Sí.

Entrevistadora: ¿Qué más es una experiencia de ocio?

\section{Frida: Experiencia, ¿sí? Experiencia es coquetear.}

\author{
Entrevistadora: ¿Coquetear es una \\ experiencia de ocio?
}

Frida: Sí.

El relato de Frida presenta el ocio como una ocupación humana, al com- prender ocupación como actividad significativa presente en el diario vivir (Zemke y Clarke, 1996). Una ocupación que ella vive sin un tiempo determinado o institucionalizado. En realidad, ella utiliza su cuerpo para gozar del ocio cuando su deseo así lo pide.

La vivencia del ocio de Frida, marcado por la búsqueda de excitación y placer, que rompe patrones al negarse a ser institucionalizado, dialoga con los elementos que la Ciencia Ocupacional plantea respecto a la ocupación: forma, función y significado (Zemke y Clark,1996). Ese diálogo, presentado por el relato de este caso, trae implicaciones para la Terapia Ocupacional como profesión que estudia y actúa en y con las ocupaciones humanas.

La forma se refiere a cómo se experimenta el ocio. En este caso, las vivencias sexuales de Frida son diversas en ambientes, pero están marcadas por el carácter desinstitucionalizado. Como función, el ocio expresa búsqueda de placer y excitación. Especialmente, de lugar de rebeldía, de mujer de y con deseos, de ¡feminidad viva! En última instancia, se puede afirmar que, para Frida, el ocio, que puede parecer algo común, en realidad es la manera de encontrarse consigo misma (Fonsêca, 2008).

Algunos textos y profesionales en Terapia Ocupacional recomiendan utilizar diversos modelos y enfoques para atender a las características partiiculares de las y los usuarios de los servicios. En este sentido, por ejemplo, en la tercera edición del Marco de trabajo para la práctica de la Terapia Ocupacional, de la Asociación Americana de Terapia Ocupacional -AOTA, el ocio es definido como "una actividad no obligatoria que 
está intrínsecamente motivada y en la que se participa durante un tiempo discrecional o libre, es decir, un tiempo no comprometido con ocupaciones obligatorias tales como trabajo, cuidado propio o dormir" (Parham y Fazio, citado por AOTA, 2014, p.22).

De otro lado, la producción científica respecto a la sexualidad humana en el área de salud, particularmente en Terapia Ocupacional, es limitada. Aún es un tema que genera vergüenza $y$, frente al cual, las y los estudiantes no reciben una preparación que les permita lidiar con los mitos, creencias y tabúes que emergen al tratar el tema en la atención profesional. En un estudio conducido por Silveira et al. (2014) respecto a la producción científica en este campo, solamente el $5 \%$ de los hallazgos se relacionaban con Terapia Ocupacional y sexualidad, lo que indica el tradicionalismo en el área. Jones et al. (2005), entre tanto, investigando la formación de terapeutas ocupacionales, identificaron que el tema de la sexualidad se trata poco, lo que genera oportunidades perdidas en la práctica profesional.

En este sentido, se valida a Almeida (2004) cuando refiere que el cuerpo es el medio para la experiencia de sí mismo y del otro, lo que en Terapia Ocupacional amplía la perspectiva profesional respecto a las personas. Ese cuerpo, que no es solamente un componente orgánico, es también objeto de cuidado para las y los terapeutas ocupacionales, es una expresión de las relaciones históricas y culturales, de experiencias y de subjetividades.

Así, comprender la sexualidad como ocio en Terapia Ocupacional, según la orientación del Marco de trabajo de la AOTA (2015), es problemático, ya que esta ocupación es considerada allí como una actividad de la vida diaria, no como ocio. Así mismo, aun cuando se mencione en el dominio de participación y la satisfacción sea un elemento señalado en su caracterización, se entiende en este artículo que el ocio no se limita a estar con otros en actividad o a la búsqueda de placer.

Por lo tanto, se opta aquí por una idea ampliada de ocio que asocie las propuestas de Elias y Dunning (1992) y los elementos anteriormente mencionados de la Ciencia Ocupacional. Este planteamiento abre posibilidades y oportunidades para reconocer los deseos respecto al propio cuerpo y la autonomía en las decisiones de las y los usuarios de servicios de salud mental atendidos o acompañados por terapeutas ocupacionales.

Se reconoce, entonces, que este artículo presenta elementos poco estudiados y que pueden aportar de manera significativa a la práctica y a la formación de terapeutas ocupacionales. Estos elementos también estimulan una visión política y transformadora del ocio en la construcción de la autonomía, la independencia y la ciudadanía. Igualmente, se destaca que este análisis fue realizado por cuatro mujeres y trata el tema del ocio de otra mujer que hace parte de una población singular (usuaria de servicios de salud mental); por lo tanto, sus hallazgos no son generalizables. Aun así, contribuye al acervo profesional relacionado con el ocio y la sexualidad.

\section{Consideraciones finales}

El ocio cumple una función en la construcción de lo cotidiano, por supuesto, no es antítesis de las actividades obligatorias, 
así que contribuye con la composición de la vida del ser humano.

Las decisiones en esa esfera pública y personal del sujeto (ocio) pueden tomarse teniendo en cuenta la satisfacción que la actividad puede proporcionar. En ese sentido, es necesario mirar más allá de los estigmas y los prejuicios sociales que tienden a controlar los comportamientos, los sentimientos y las expresiones en un mundo de sujetos diversos.

Al analizar el punto de vista de Frida sobre su propio ocio desde la perspectiva de Norbert Elias y Erick Dunning, que contempla las categorías placer y excitación, fue posible entender el significado, la forma y la función de la ocupación ocio en la vida de esta habitante de una residencia terapéutica. El diálogo entre dicha perspectiva y los elementos de la Ciencia de la Ocupación trae posibilidades de actuación distintas para la Terapia Ocupacional. Posibilidades que reconozcan las diferentes maneras de vivir el ocio y de valorar sus sutilezas, traducidas en forma, función y significado, elementos con los cuales las y los terapeutas ocupacionales pueden desarrollar acciones con las personas, en beneficio de una vida placentera, excitante y saludable.

De este modo, Frida, desde sus vivencias, permite entender el ocio como organizador de una nueva cotidianidad, la de una mujer en un nuevo escenario de la vida; también, ofrece un punto de reflexión sobre otras maneras de ser moradora y de habitar en la sociedad.

\section{Referencias}

Almeida, M. V. M. (2004). Corpo e arte em terapia ocupacional. Enelivros.

Asociación Americana de Terapia Ocupacional [AOTA] (2014). Occupational Therapy Practice Framework: Domain and Process (3rd Edition). American Journal of Occupational Therapy, 68 (sup.1), 1-48. https://doi.org/10.5014/ ajot.2014.682006

Ministerio de Salud, Brasil (2000, 11 de febrero). Ordenanza Ministerial 106. Crea los Servicios Residenciales Terapéuticos en Salud Mental, en el ámbito del Sistema Único de Salud, para la atención de personas con trastornos mentales. http://www.saude.gov. br/images/pdf/2015/marco/10/PORTARIA-106-11-FEVEREIRO-2000.pdf

Brito, M.A.M. y Dimenstein, M. (2008). Contornando as grades do manicômio: histórias de resistências esculpidas na instituição total. Aletheia, (28), 188-203. http://pepsic. bvsalud.org/scielo.php?script=sci arttext\&pid=S1413-03942008000200015\&ln$\mathrm{g}=\mathrm{pt} \& \mathrm{t} \operatorname{lng}=\mathrm{pt}$.

Covino, A. M. (2007). O cotidiano nos espaços de morar e habitar em saúde mental [tesis de maestría, Universidade de São Paulo]. Bibliothèque Numérique USP. https://teses. usp.br/teses/disponiveis/7/7134/tde19122007-162841/fr.php

Dias, E., Duarte, Y., Almeida, M. y Lebrão, M. (2011). Caracterização das atividades avançadas de vida diária (AAVDS): um estudo de revisão. Revista de Terapia Ocupacional da Universidade de São Paulo, 22(1), 45-51. https://doi.org/10.11606/ issn.2238-6149.v22i1p45-51 
Elias, N. y Dunning, E. (1992). Memória e Sociedade a Busca da Excitação. Difel.

Fonsêca, M.A. (2008). A prática do terapeuta ocupacional em saúde mental a partir de uma perspectiva não excludente e de respeito as diferenças. En A. Drummond y M. Rezende (eds.), Intervenções da terapia ocupacional (pp.71-94). UFMG.

Jones, M.K., Weerakoon, P. y Pynor, R.A. (2005). Survey of occupational therapy student's attitudes towards sexual issues in clinical practice. Occupational Therapy International, 12 (2), 95-106. https://doi.org/10.1002/oti.18

Marazina, I.V. (2000). FEBEM: Os descaminhos do pai. Estilos da Clinica, 5(8), 190-199. http://pepsic. bvsalud.org/scielo.php?script=sci arttext\&pid=S1415-71282000000100017\&ln$\mathrm{g}=\mathrm{pt} \& \mathrm{t} \operatorname{lng}=\mathrm{pt}$

Orsi, V. (2011). Tabu e preconceito linguístico. Revista Virtual de Estudos da Linguagem, 9(17), 334-348. http://hdl.handle.net/11449/122427

Queiroz, A.G. (2015). Não tô boa. Preciso passear!: o lazer de moradores de dois serviços residenciais terapêuticos de Belo Horizonte [tesis de maestría, Universidade Federal de Minas Gerais]. Repositório Institucional da UFMG. http:// hdl.handle.net/1843/EEFF-BB6FCD

Salles, M.M. y Matusukura, T.S. (2013). Estudo de revisão sistemática sobre o uso do conceito de cotidiano no campo da terapia ocupacional no Brasil. Cadernos Brasileiros de Terapia Ocupacional, 21(2), 265-273. http://doi.org/10.4322/ cto. 2013.028
Silveira, G.F., Wittkopf, P.G., Sperandio, F.F. y Pivetta, H.M.F. (2014) Produção científica da área da saúde sobre a sexualidade humana. Saúde e Sociedade, 23(1), 302-312. https://doi.org/10.1590/S010412902014000100024.

Zemke, R. y Clark, F. (1996). Occupational science: The evolving discipline. Davis. 